\title{
Content type revamp
}

\author{
We've made some recent updates to our various content type offerings at Nature Methods. Here is a cheat sheet.
}

A rticle or Brief Communication? Brief Communication or Correspondence? Review or Perspective? Authors might agonize over which content type to choose to submit their work to Nature Methods. We hope we can help remove some confusion and stress by stating that the selection of content type is not important at the initial submission stage. Most of the time our editorial team can immediately judge whether a manuscript would be better suited for a different content type; other times we like to get feedback from reviewers before making this call. Rest assured, if the paper is a good fit for Nature Methods, we will find the appropriate content type fit.

Additionally, although we encourage authors to familiarize themselves with our Guide to Authors, it is not necessary at the initial submission stage to follow publication formatting standards. We will never reject an interesting paper simply because it is not formatted exactly right for publication, nor because the English isn't perfect. There is plenty of time to address these issues during the publication process, and we take pride in helping authors present their work in Nature Methods in a way that is appealing, readable and scientifically accurate.

The Nature Research editorial teams have been working together to harmonize the definitions of our content type offerings. In light of this, regular readers of Nature Methods may notice a few changes. Here we provide a short, handy guide to our content types and describe some recent updates.

We have several primary research content types. Such manuscripts are selected by the editorial team for their novelty, potential interest and advance, and are subject to rigorous peer review.

An Article is our main content type for presenting novel methods. We expect Articles to provide a full technical description and strong validation of the method to demonstrate its performance, reproducibility, general applicability and potential for discovering new biology. (Note that we don't require reports of novel biological findings, but this is always a plus!)

Over the years, our Brief

Communications have bloated in length, blurring their distinction from Articles. We are now restricting Brief Communications to two- or three-page reports describing exciting yet preliminary new methods, highly practical tweaks to existing methods or tools, software platforms, resources of broad interest, and technical critiques of widely used methodologies.

A Resource may present a collection of tools or datasets of broad utility, including comprehensive characterization or extensive use cases.

An Analysis presents comprehensive performance comparisons of established, related methods or tools, of key importance to a field of research. Many Analyses originate from multiple lab groups, which helps avoid bias favoring a lab's preferred method. In the near future, we hope to offer a new approach for peer review of Analyses, enabling reviewers to give authors detailed feedback on experimental design plans before any experimental work is completed. We welcome presubmission inquiries from authors who are interested in working with our editorial team to develop and test this new workflow.

Our new Matters Arising content type is dedicated to publishing constructive comments on Nature Methods papers. If authors wish to highlight technical flaws or limitations of a popular method (of course, supported by strong data and analysis), such a paper should be submitted as a Brief Communication.

Correspondence has previously been a catch-all content type for many types of manuscripts. Following in the footsteps of our other Nature Research colleagues, we are now no longer publishing Correspondence describing new methods or reporting research data (including as Supplementary Information). Such manuscripts will need to meet our standards for Brief Communications to be considered for publication.

Correspondence will be strictly limited to short announcements of broadly interesting tools, resources, initiatives, news and opinions. Correspondence are peer reviewed at the discretion of the editorial team.

We also have several review-type formats.

A Review is a well-balanced, in-depth survey of the literature on an established technology or class of methods. In contrast, a Perspective may be written on a newer technology, such as one developed mainly in a few labs, and may contain strong author opinions. The differences in their formatting are minor. Our editorial team commissions some Reviews and Perspectives, but we also welcome proposals via presubmission inquiries. Reviews and Perspectives are always peer reviewed.
A Comment is an opinion piece on a topic of broad interest. Authors may describe their personal perspectives on a particular methodology or field of research, discuss issues that affect their communities, or propose guidelines for performing experiments or data reporting and sharing. Some Comments we publish are commissioned, but we also welcome proposals via presubmission inquiries. Comments may be peer reviewed at the discretion of the editorial team.

A News and Views highlights a Nature Methods paper, places it into context with work that has come before, and discusses future developments and applications as well as limitations. Rarely, we also highlight methods published in other journals. Such pieces are nearly always commissioned, and are not peer reviewed.

We also publish several types of content that are generated by the editorial team, journalists, and regular freelancers.

Editorials represent the journal opinion on current events; we also highlight journal policy and initiatives, and promote best practices in research publishing.

Research Highlights feature recent methods and tools published in journals other than Nature Methods. Our regular readers may notice that as of this month we are no longer publishing shorter "In Brief" pieces. Instead, we will be highlighting interesting methodological developments via the Protocols and Methods Community page and via Twitter(@naturemethods).

We have two types of journalistic content. The Author File is a profile of an author of a Nature Methods paper published in the same issue. The Technology Feature checks in with researchers working in a field of interest to Nature Methods.

Points of Significance is a non-peerreviewed column written by regular freelancers Martin Krzywinski and Naomi Altman. This column is intended to educate readers about core statistical approaches commonly used in biological research.

We hope our readers find this diversity of content to be interesting and useful to daily life in the lab. As always, we welcome your feedback!

Published online: 31 July 2020 https://doi.org/10.1038/s41592-020-0927-4 